\title{
Ambulatory management of primary spontaneous pneumothorax: the jury is still out
}

\section{To the Editor}

Pneumothorax is a commonly encountered condition in clinical practice. The drainage remains an essential treatment for most patients with secondary spontaneous pneumothorax; however, the ideal treatment of primary spontaneous pneumothorax still remains unclear. The British Thoracic Society guidelines suggest the use of initial aspiration with the help of a syringe and cannula [1]. As a large proportion will still end up requiring a chest tube insertion, better ways of management are being investigated. One such method is the use of a small valved device which can be left in place and allows the patient to remain ambulatory (Figure 1). In a recent issue of The Lancet, Hallifax et al. have published the results of randomized controlled trial comparing the ambulatory device with the standard care for primary spontaneous pneumothorax (PSP) [2]. Although the authors have concluded that ambulatory management of PSP is a reasonable and preferable option, there are few issues which need discussion.

The primary outcome chosen for the study was the duration of hospital stay, which itself is not ideal. The primary goal of PSP treatment is symptom relief and recurrence prevention. Using an ambulatory device is likely to reduce the hospitalization in settings where patients with chest drains are routinely hospitalized. The time to the symptom or radiological resolution, or patient comfort, would be more clinically relevant outcomes. Secondly, the chest drain removal and discharge criteria were not protocolized for the control group, which is a source of bias in pri- mary outcome assessment. Around one-third of patients in the standard arm could be managed with single aspiration; however, all patients in the ambulatory arm underwent device insertion, which could have been avoided in a significant proportion by simple aspiration. A solution for this confounder would be randomization after the failure of simple aspiration. Serious adverse

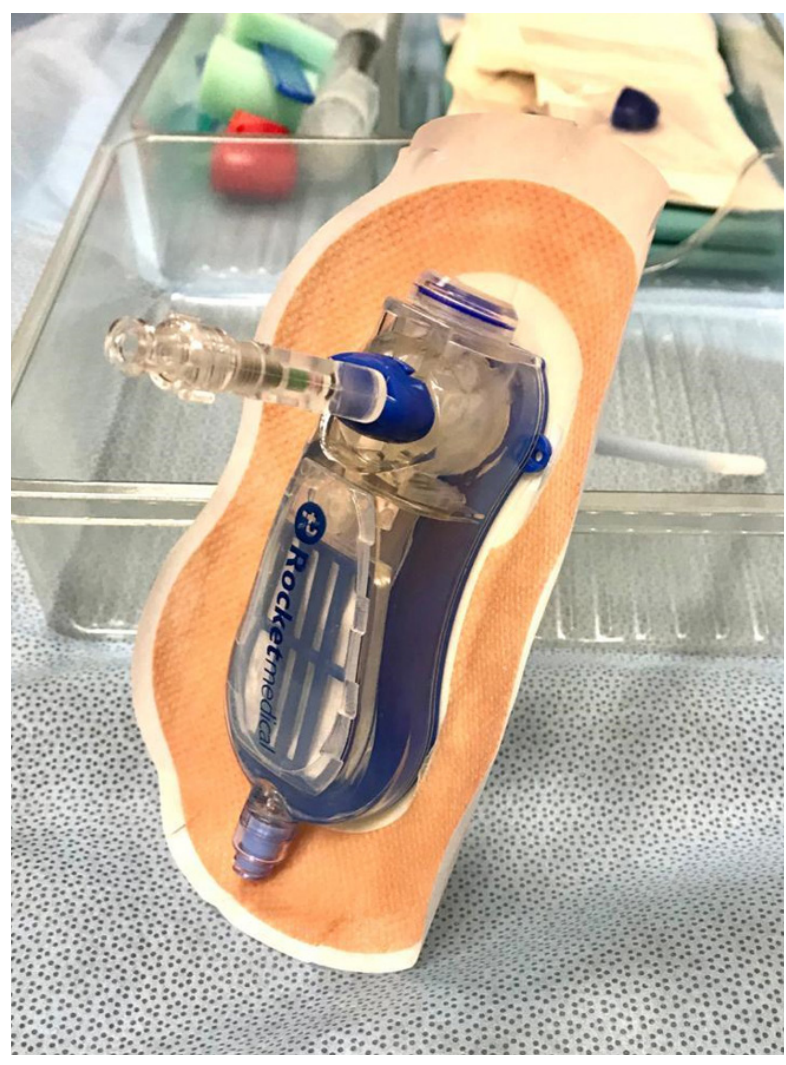

Figure 1. The valved ambulatory device available for the use for drainage of pneumothorax 
events related to the device were high, necessitating a strict follow-up and daily review. Although it is suggested to use a written action plan for the same, this may not be feasible in all settings, especially in most developing countries. This suggests that results may not be applicable to a large proportion of individuals globally. Given these concerns, we suggest that the ambulatory device should be used with a constant vigil only in a selected group of patients consenting for regular follow-up and monitoring.

\section{Conflict of interest}

None declared.

\section{References:}

1. MacDuff A, Arnold A, Harvey J. Management of spontaneous pneumothorax: British Thoracic Society Pleural Disease Guideline 2010. Thorax. 2010; 65 Suppl 2: ii18-ii31, doi: 10.1136/thx.2010.136986, indexed in Pubmed: 20696690.

2. Hallifax RJ, McKeown E, Sivakumar P, et al. Ambulatory management of primary spontaneous pneumothorax: an open-label, randomised controlled trial. Lancet. 2020; 396(10243): 39-49, doi: 10.1016/S0140-6736(20)31043-6, indexed in Pubmed: $\underline{32622394}$. 